\title{
TCM coding of PPM based modulations for Infrared WLAN's impaired by ISI
}

\author{
Rui Alves, Atílio Gameiro
}

\begin{abstract}
In this communication we consider the use of PPM based modulation methods, such as the hybrid modulation method called Amplitude and Pulse-Position Modulation (APPM) and Overlapping Pulse-Position Modulation (OPPM) to improve the performance of infrared WLAN's, by the use of Trellis-Coded Modulation (TCM) codes. We describe the best code search and results, which demonstrate that, even with trellis codes of moderate complexity, non-negligible coding gains can be obtained without bandwidth expansion. Monte Carlo simulations have been done to compare uncoded PPM against APPM and OPPM TCM coded systems performances, on ceiling-bounce channel models for various values of delay spread. Although our codes were derived for the AWGN channel without Inter-Symbol Interference (ISI) awareness, their behavior in multipath dispersion channels is quite effective.
\end{abstract}

Index Terms - Infrared wireless communications, Pulse Position Modulation, Trellis-Coded Modulation, InterSymbol Interference.

\section{INTRODUCTION}

$\mathrm{T}$ HERE has been in recent years, considerable work on infrared links for WLAN's [1]. Standardization work groups such as the IEEE 802.11[2] and the Infrared Data Association (IrDA) [3], cover both point-to-point systems and local area networks, showing the vast potential of the infrared technology. Its key advantages over radio technology rely on the virtually unlimited, unregulated bandwidth, available worldwide, and on its inherent security characteristics granted by the natural confinement of infrared signals inside closed rooms.

The goal of infrared link designers is to achieve reliable indoor point-to-point connectivity between any devices in a network. Though, the indoor office environment is populated with unregulated sources of light, creating a variable and noisy environment, which make the optical link design to be a difficult task. There are a large number of issues being focused by the infrared research. In this communication we address the modulation and coding issues jointly, analyzing and comparing the systems: uncoded PPM and Trellis-Coded Modulation (TCM) using PPM based modulation techniques. The PPM based modulation techniques we consider are:

- APPM (Amplitude and Pulse-Position Modulation) [4], a hybrid modulation where information is conveyed both in the amplitude and the position of one pulse.

- OPPM (Overlapped Pulse Position Modulation) [5] where the symbols contain a pulse transmitted in non-orthogonal positions, i.e. allowing some overlapping between pulses of different symbols.

These modulation schemes mainly preserve the high average-power efficiency of PPM, holding larger alphabet sizes without a significant bandwidth expansion [6]. This makes both APPM and OPPM suitable schemes to apply TCM techniques, and then to improve the performance of wireless infrared communication systems.

The communication is outlined as follows. The next section presents the system model and the modulation schemes we will be dealing with. Section 3 describe the computational search for optimum TCM codes with some APPM and OPPM schemes, present the codes found for some code memory orders and the respective asymptotic coding gains relatively to the uncoded 2-PPM system, in the ISI absence scenario. In section 4 we report the Monte Carlo simulations made to verify the accuracy of our analysis, and to evaluate the performance of our proposed codes when running within multipath ISI channels. Finally in section 5 the main conclusions of this work are pointed to.

\section{SYSTEM MODEL}

Fig. 1 shows the block diagram of the system we are concerned with, where the input data sequence $\left\{a_{j}\right\}$ is encoded, modulated and converted to an optical power signal $X(t)$. The transmission multipath channel is modeled by a channel impulse response $h(t)$ (constant to a given configuration of transmitter, receiver, and intervening reflectors), and an AWGN source $N(t)$ independent of $X(t)$ with power spectral density $N_{\mathrm{o}}$. At the receiver the channel adaptation is made through a WMF, which consists in a unitenergy matched filter, a sampler and a noise-whitening filter. After convenient zero-forcing equalization the MLSD is made by the Viterbi TCM decoder. Hence, the incoming photocurrent $Z(t)$ gives the output data sequence $\left\{\hat{a}_{j}\right\}$, which is a $\left\{a_{j}\right\}$ sequence replica except for some positions where the decoder was not able to correct channel errors. In the case of TCM coding systems the (de)coding and (de)modulation operations must be combined.

In order to compare systems applying different modulation schemes, coded or not, the following electrical SNR definition is considered:

$$
\mathrm{SNR}=\frac{2 P_{a v}^{2} \Re^{2} T_{c}}{N_{0}}
$$

where $P_{a v}$ and $T_{c}$ are, respectively, the optical average power and the chip duration for the modulation involved, and $\mathfrak{R}$ is the photodetector responsivity. 

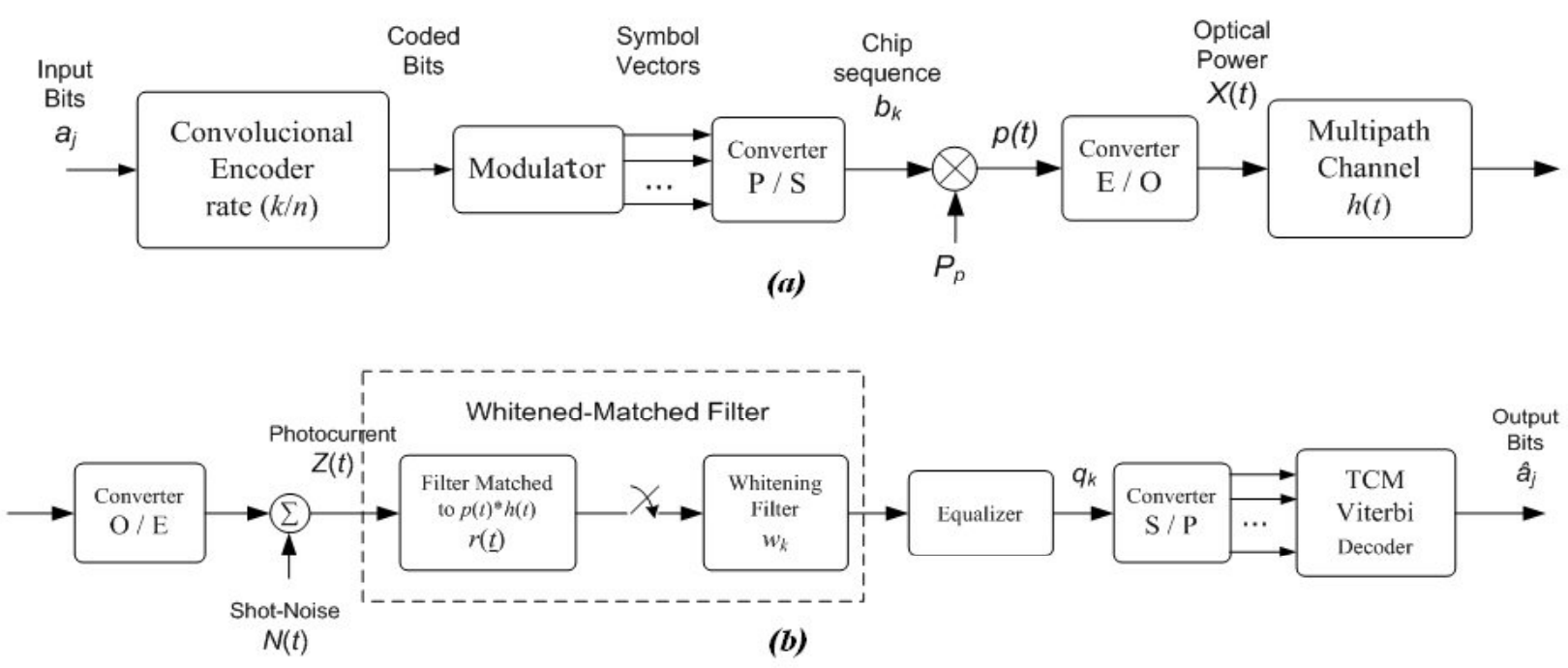

Fig. 1. Block diagram of the trellis-coded transmission system over a multipath channel.

(a) Emitter and channel (b) Receiver with WMF and decoder.

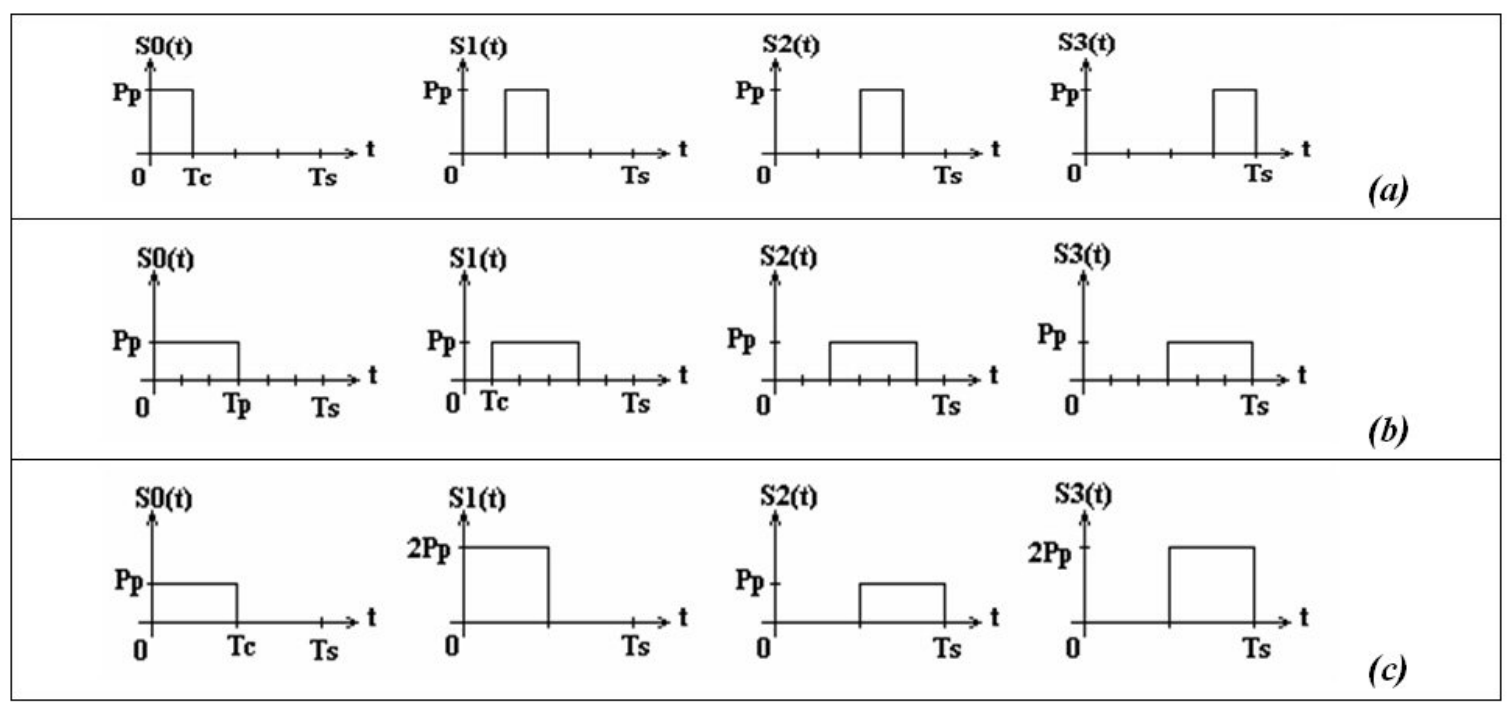

Fig. 2. Transmitted waveforms for schemes with alphabet size 4: (a) 4-PPM (b) (6 3) OPPM and (c) 2×2-APPM.

The modulation formats we consider are PPM, APPM and OPPM. Figure 2 represents the alphabet symbols from some representative schemes.

In $M$-PPM modulations, each word of $k$ bits is mapped into one of $M=2^{k}$ symbols and transmitted to the channel. The symbol interval is divided into $M$ chips, and each symbol uses a different chip to transmit a pulse. Thus, the $M$ possible waveforms are: $p_{k}(t)=P_{p} p_{T_{c}}\left(t-i T_{c}\right)$ with $i \in\{0,1, \ldots, M-1\}$, and $p_{T_{c}}(t)$ being a rectangular pulse with unit amplitude and chip duration $T_{c}=T_{S} / M$. The average power is related with the peak power by $P_{a v}=P_{p} / M$.

In an $A \times M$-APPM symbol there are $M$ chips of $T_{c}$ duration, like for $M$-PPM, even so the pulse may take one of $A$ possible amplitude values. Therefore, the $A \times M$ different symbol waveforms are: $p_{k}(t)=j P_{p} p_{T_{c}}\left(t-i T_{c}\right)$, with $j \in\{1,2, \ldots, A\}$, and $i \in\{0,1, \ldots, M-1\}$. The average power relates with the peak power by $P_{a v}=P_{p}(1+A) / 2 M$.

For $(n w)$ Overlapping PPM [5] schemes, the symbol interval $T_{s}$ is divided into $n$ chips of equal duration. The information is conveyed on the pulse position. The pulse has a duration $T_{p}=w T_{c}$, and can start in one of the $M$ instants $t_{k}=(k-1) T_{c}$ with $k=1,2, \ldots, M$, being $t_{1}=0$ the symbol interval beginning. It is clear that $M$ is related to $n$ and $w$ by: $M=n-w+1$. Thus, the $M$ waveforms allowed for $M$-OPPM symbols are: $p_{k}(t)=P_{p} p_{T_{p}}\left(t-i T_{c}\right)$ with $i \in\{0,1, \ldots, M-1\}$, and the chip duration $T_{c}=T_{s} / n$. The relation between average and peak powers is $P_{a v}=P_{p} w / n$. Efficient OPPM schemes require the selection of ( $n w)$ pairs leading $M$ to be a power of 
TABLE I

OPTIMUM TCM CODES AND ACHIEVED FREE DISTANCES FOR APPM AND OPPM MODULATIONS.

$(*)$ AND $(* *)$ MEAN IMPRACTICAL AND INCOMPLETE SEARCH, RESPECTIVELY.

\begin{tabular}{|c|c|c|c|c|c|c|c|c|c|}
\hline \multirow{2}{*}{ Modulation } & \multicolumn{2}{|c|}{ Code } & \multicolumn{7}{|c|}{ Encoder Memory Order } \\
\hline & Rate & Defin.: & 2 & 3 & 4 & 5 & 6 & 8 & 10 \\
\hline \multirow{2}{*}{$2 \times 2-\mathrm{APPM}$} & \multirow{2}{*}{$1 / 2$} & $d_{\text {free }}^{2}$ & 12 & 14 & 16 & 17 & 19 & 22 & 26 \\
\hline & & $h^{0} ; h^{1}$ & $7 ; 2$ & $15 ; 2$ & $31 ; 02$ & $45 ; 12$ & $187 ; 32$ & $607 ; 20$ & $3427 ; 0274$ \\
\hline \multirow{2}{*}{$4 \times 2$-АРPM } & \multirow{2}{*}{$1 / 3$} & $d_{\text {free }}^{2}$ & 31 & 36 & 42 & 46 & 53 & 66 & $(*)$ \\
\hline & & $h^{0} ; \mathrm{z}^{1} ; \mathrm{z}^{2}$ & $3 ; 2 ; 5$ & $6 ; 02 ; 17$ & $16 ; 12 ; 27$ & $36 ; 16 ; 63$ & $71 ; 056 ; 135$ & $375 ; 236 ; 545$ & $(*)$ \\
\hline \multirow{2}{*}{$2 \times 4-\mathrm{APPM}$} & \multirow{2}{*}{$2 / 3$} & $d_{\text {free }}^{2}$ & 6 & 7 & 8 & 10 & 11 & 13 & 15 \\
\hline & & $h^{0} ; h^{1} ; h^{2}$ & $7 ; 2 ; 1$ & $13 ; 1 ; 7$ & $27 ; 1 ; 4$ & $65 ; 02 ; 20$ & $147 ; 6 ; 70$ & $661 ; 004 ; 376$ & $3035 ; 0002 ; 1155$ \\
\hline \multirow{5}{*}{ 4×4-АРPM } & \multirow{5}{*}{$2 / 4$} & $\overline{d_{\text {free }}^{2}}$ & 31 & 38 & 42 & 48 & 54 & $(*)$ & $(*)$ \\
\hline & & $h^{11} ; h^{12}$ & $2 ; 1$ & $4 ; 0$ & $4 ; 4$ & $10 ; 10$ & $10 ; 10$ & $(*)$ & $(*)$ \\
\hline & & $h^{21} ; h^{22}$ & $2 ; 3$ & $2 ; 1$ & $4 ; 7$ & $7 ; 4$ & $02 ; 05$ & $(*)$ & $(*)$ \\
\hline & & $\mathrm{z}^{11} ; \mathrm{z}^{12}$ & $2 ; 0$ & $0 ; 0$ & $0 ; 6$ & $01 ; 03$ & $10 ; 10$ & $(*)$ & $(*)$ \\
\hline & & $\mathrm{z}^{21} ; \mathrm{z}^{22}$ & $2 ; 3$ & $1 ; 3$ & $3 ; 1$ & $3 ; 7$ & $05 ; 17$ & $(*)$ & $(*)$ \\
\hline \multirow{2}{*}{ (5 2)-OPPM } & \multirow{6}{*}{$1 / 2$} & $d_{\text {free }}^{2}$ & 4.00 & 4.80 & 5.60 & 6.40 & 8.00 & 9.60 & 11.2 \\
\hline & & $h^{0} ; h^{1}$ & $5 ; 2$ & $15 ; 2$ & $31 ; 02$ & $55 ; 02$ & $117 ; 42$ & $515 ; 072$ & $3453 ; 0246$ \\
\hline \multirow{2}{*}{ (6 3)-OPPM } & & $d_{\text {free }}^{2}$ & 3.33 & 4.00 & 4.67 & 5.33 & 6.67 & 8.0 & 9.33 \\
\hline & & $h^{0} ; h^{1}$ & $5 ; 2$ & $15 ; 2$ & $31 ; 02$ & $55 ; 02$ & $117 ; 42$ & $515 ; 072$ & $3453 ; 0246$ \\
\hline \multirow{2}{*}{ (7 4)-OPPM } & & $d_{\text {free }}^{2}$ & 2.86 & 3.43 & 4.00 & 4.57 & 5.71 & 6.86 & 8.00 \\
\hline & & $h^{0} ; h^{1}$ & $5 ; 2$ & $15 ; 2$ & $31 ; 02$ & $55 ; 02$ & $117 ; 42$ & $515 ; 072$ & $3453 ; 0246$ \\
\hline \multirow{2}{*}{ (9 2)-OPPM } & \multirow{6}{*}{$2 / 3$} & $\overline{d_{\text {free }}^{2}}$ & 2.67 & 3.56 & 4.44 & 5.33 & 5.33 & 7.11 & 8.00 \\
\hline & & $h^{0} ; h^{1} ; h^{2}$ & $5 ; 1 ; 2$ & $11 ; 1 ; 2$ & $21 ; 3 ; 10$ & $45 ; 7 ; 24$ & $103 ; 1 ; 22$ & $523 ; 151 ; 344$ & $(* *)$ \\
\hline \multirow{2}{*}{ (10 3)-OPPM } & & $d_{\text {free }}^{2}$ & 3.20 & 4.00 & 4.80 & 4.80 & 5.60 & 7.20 & 8.00 \\
\hline & & $h^{0} ; h^{1} ; h^{2}$ & $7 ; 1 ; 3$ & $11 ; 2 ; 4$ & $23 ; 4 ; 16$ & $63 ; 1 ; 14$ & $143 ; 1 ; 24$ & $667 ; 112 ; 314$ & $(* *)$ \\
\hline \multirow{2}{*}{ (11 4)-OPPM } & & $d_{\text {free }}^{2}$ & 2.18 & 2.91 & 4.36 & 4.36 & 5.09 & 6.55 & 7.27 \\
\hline & & $h^{0} ; h^{1} ; h^{2}$ & $5 ; 1 ; 2$ & $11 ; 1 ; 2$ & $21 ; 1 ; 2$ & $73 ; 1 ; 6$ & $137 ; 5 ; 76$ & $453 ; 275 ; 154$ & $(* *)$ \\
\hline
\end{tabular}

2, such as: (5 2), (6 3) and (7 4) for $M=4$, or (9 2), (10 3) for $M=8$.

\section{TCM CODES SEARCH FOR APPM AND OPPM}

The classical separation of coding and modulation requires some bandwidth expansion, what may be unacceptable when the channel is bandwidth constrained. To illustrate ideas let us consider the use of PPM with a rate-1/2 convolutional code. In the absence of coding, the chip duration of a $2^{k}$-PPM pulse is related to the bit duration through:

$$
T_{c}=\frac{k}{2^{k}} \cdot T_{b},
$$

while with the use of a $1 / 2$ convolutional code this goes to:

$$
T_{c}=\frac{k}{2 \cdot 2^{k}} \cdot T_{b} .
$$

Considering the first order approximation of PPM bandwidth proportional to the chip rate inverse, in a bandwidth limited system the coding introduction will require a PPM order reduction. For instance, we should go from uncoded 16-PPM to coded 4-PPM or from uncoded 64-PPM to coded 16-PPM, to prevent a bandwidth expansion. Asymptotically this represents a power penalty through the reduction of the set dimensionality of approximately 3 optical $\mathrm{dB}$, which demand a minimal $3 \mathrm{~dB}$ coding gain from the convolutional code with hard decoding.

In these cases, one should combine the modulation and coding schemes accordingly TCM. It was already pointed out at [6], that both APPM and OPPM are suitable schemes to augment the alphabet size of PPM to apply TCM techniques without bandwidth expansion, and then to improve the performance of wireless infrared communication systems.

So that their coding merits can be assessed, we must search the TCM codes that maximize the asymptotic coding gains. For the desired infrared link applications working on high SNRs and having low error-probabilities, the asymptotic coding gain is a good approximation to the real coding gain achieved by TCM codes. At this stage, we do not consider the ISI effects due to multipath channel operation, i.e. the best coding search is performed to carry on the simplest AWGN channel. In that case, the asymptotic coding gain of a TCM coded modulation scheme, relatively to uncoded 2-PPM transmission is computed by (4), where $d_{\text {free }}^{2}$ is the squared minimum Euclidean distance between all coded sequences, 
TABLE II

ASYMPTOTIC CODING GAINS FOR THE BEST TCM CODES FOUND WITH APPM AND OPPM MODULATIONS RELATIVELY TO 2-PPM.

(*) MEANS AN IMPRACTICAL SEARCH.

\begin{tabular}{|c|c|c|c|c|c|c|c|c|c|}
\hline \multirow{3}{*}{ Modulation } & \multirow{3}{*}{$\boldsymbol{G}_{c o d}\left(\mathrm{~dB}_{\text {electric }}\right)$} & \multirow{3}{*}{$\begin{array}{c}\text { Encoder } \\
\text { Rate }\end{array}$} & \multicolumn{7}{|c|}{ 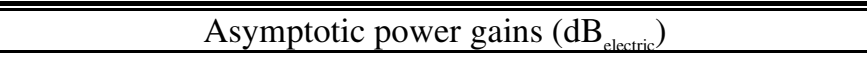 } \\
\hline & & & \multicolumn{7}{|c|}{ Encoder memory order } \\
\hline & & & 2 & 3 & 4 & 5 & 6 & 8 & 10 \\
\hline $2 \times 2-\mathrm{APPM}$ & $10 \log _{10}\left(\frac{2}{9} d_{\text {free }}^{2}\right)$ & $1 / 2$ & 4.26 & 4.93 & 5.51 & 5.77 & 6.26 & 6.89 & 7.62 \\
\hline $4 \times 2-\mathrm{APPM}$ & $10 \log _{10}\left(\frac{2}{25} d_{\text {free }}^{2}\right)$ & $1 / 3$ & 3.94 & 4.59 & 5.26 & 5.66 & 6.27 & 7.22 & $(*)$ \\
\hline 2×4-APPM & $10 \log _{10}\left(\frac{8}{9} d_{\text {free }}^{2}\right)$ & $2 / 3$ & 7.27 & 7.94 & 8.52 & 9.49 & 9.90 & 10.63 & 11.25 \\
\hline 4×4-APPM & $10 \log _{10}\left(\frac{8}{25} d_{\text {free }}^{2}\right)$ & $2 / 4$ & 9.96 & 10.85 & 11.28 & 11.86 & 12.37 & $(*)$ & $(*)$ \\
\hline (5 2)-OPPM & \multirow{6}{*}{$10 \log _{10}\left(\frac{n^{2}}{8 w^{2}} d_{\text {free }}^{2}\right.$} & \multirow{3}{*}{$1 / 2$} & 4.95 & 5.74 & 6.41 & 6.99 & 7.96 & 8.75 & 9.42 \\
\hline (6 3)-OPPM & & & 3.01 & 3.80 & 4.47 & 5.05 & 6.02 & 6.81 & 7.48 \\
\hline (7 4)-OPPM & & & 1.85 & 2.64 & 3.31 & 3.89 & 4.86 & 5.65 & 6.32 \\
\hline (9 2)-OPPM & & \multirow{3}{*}{$2 / 3$} & 8.30 & 9.55 & 10.5 & 11.3 & 11.3 & 12.6 & 13.1 \\
\hline (10 3)-OPPM & & & 6.48 & 7.44 & 8.24 & 8.24 & 8.91 & 10.0 & 10.46 \\
\hline (11 4)-OPPM & & & 3.14 & 4.39 & 6.15 & 6.15 & 6.82 & 7.92 & 8.37 \\
\hline
\end{tabular}

$\bar{P}_{c o d}$ the mean power of the constellation signals that constitute the modulation alphabet, $d_{u n c}^{2}$ the squared minimum Euclidean distance between 2-PPM symbols and $\bar{P}_{\text {unc }}$ the 2-PPM alphabet mean power. The 2-PPM pulse's amplitude normalization to 1 , leads to $\bar{P}_{u n c}=1 / 2$ and $d_{u n c}^{2}=2$.

$$
G_{c o d}=10 \log \left[\frac{\left(d_{\text {free }}^{2} / \bar{P}_{c o d}\right)}{\left(d_{\text {unc }}^{2} / \bar{P}_{\text {unc }}\right)}\right] \quad(d B)
$$

So, from (4) we can conclude that the asymptotic coding gain maximization for a given modulation scheme, requires to find out the convolutional encoder with maximal $d_{\text {free }}^{2}$. This is the criterion used to find out the best TCM codes, attained by performing an exhaustive computational search.

Efficient TCM codes with the proposed APPM and OPPM schemes require $1 / 2,2 / 3,1 / 3$ and $2 / 4$ rate encoders. For more details on used coding structures and their polynomial definitions, please refer to [6]. After exhaustive inspection, we noted all considered TCM codes, using any APPM or OPPM modulation schemes, to fit in the symmetry class of quasiregular codes (QRC or ZWC) [7]. Consequently, the GUZWA algorithm [7] had been used to proceed with the respective $d_{\text {free }}^{2}$ evaluation.

Table 1 presents the results of this search: the best free distance attained and one of the best codes found, for each modulation and for memory orders $(v)$ between 2 and 10 . The polynomials defining the different encoders use the octal notation as followed by Pietrobon in [8].

Table 2 contains the asymptotic power gains expressions, derived through (4), for each TCM system with $A \times M$-APPM and ( $n$ w)-OPPM schemes relatively to uncoded 2-PPM, as well as the achieved values for the searched best codes. We can conclude that quite appreciable gains can be obtained with convolutional encoders of moderate to low memory.

Once got the best TCM codes with APPM and OPPM schemes, a question arose: how do these codes perform at the presence of ISI in multipath channels? In order to find out an answer, next section presents some simulation results.

\section{Simulation Results}

Monte Carlo simulations have been done to evaluate uncoded PPM systems, APPM and OPPM TCM coded systems performances, on ceiling-bounce channel models [9] with delay spread per bit duration ratios $\tau_{\text {bit }}$ equal to $0,0.06$ and 0.2. Due to the prohibited times required to simulate some TCM coded systems with higher $v$ values, only the ones with $v$ values up to 6 could be simulated.

Both figures 3 and 4 refer to the ISI absence case, where $\tau_{\mathrm{bit}}=0$. The former one presents the simulated Bit-Error Rate (BER) curves for the uncoded 2- and 4-PPM, and the TCM coded 2×4-APPM, 4×4-APPM, (5 2) OPPM and (9 2) OPPM, in all cases with $v=6$, which with accurate closeness confirms expected asymptotic coding gains. The latter one shows, for uncoded 2- and 4-PPM and for APPM TCM coded systems, the SNR requirements to achieve a $\mathrm{BER}=10^{-9}$ performance, and plots the respective channel capacities whose asymptotic values are $2 \mathrm{bit} / \mathrm{Tsymbol}$ for uncoded 4-PPM and TCM coded $2 \times 4$ - and $4 \times 4$-APPM, and $1 \mathrm{bit} / \mathrm{Tsymbol}$ for the remaining ones. As one might expect, we can conclude that TCM coding brings the performance quite close to the channel capacity. 


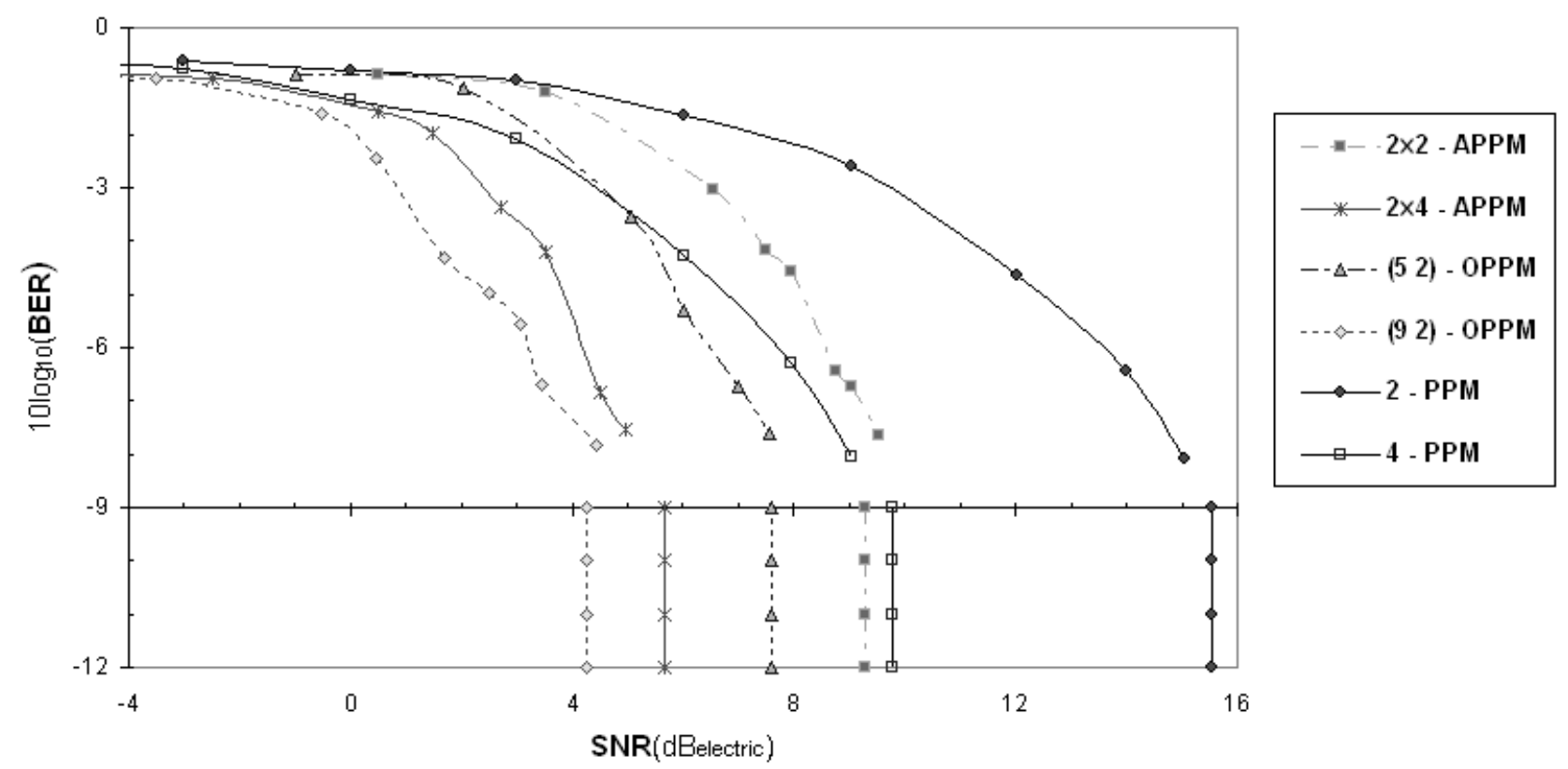

Fig. 3. Comparison of simulated performances for the systems: uncoded PPM formats and TCM coded with APPM and OPPM schemes. At the bottom side the asymptotic performances are marked.

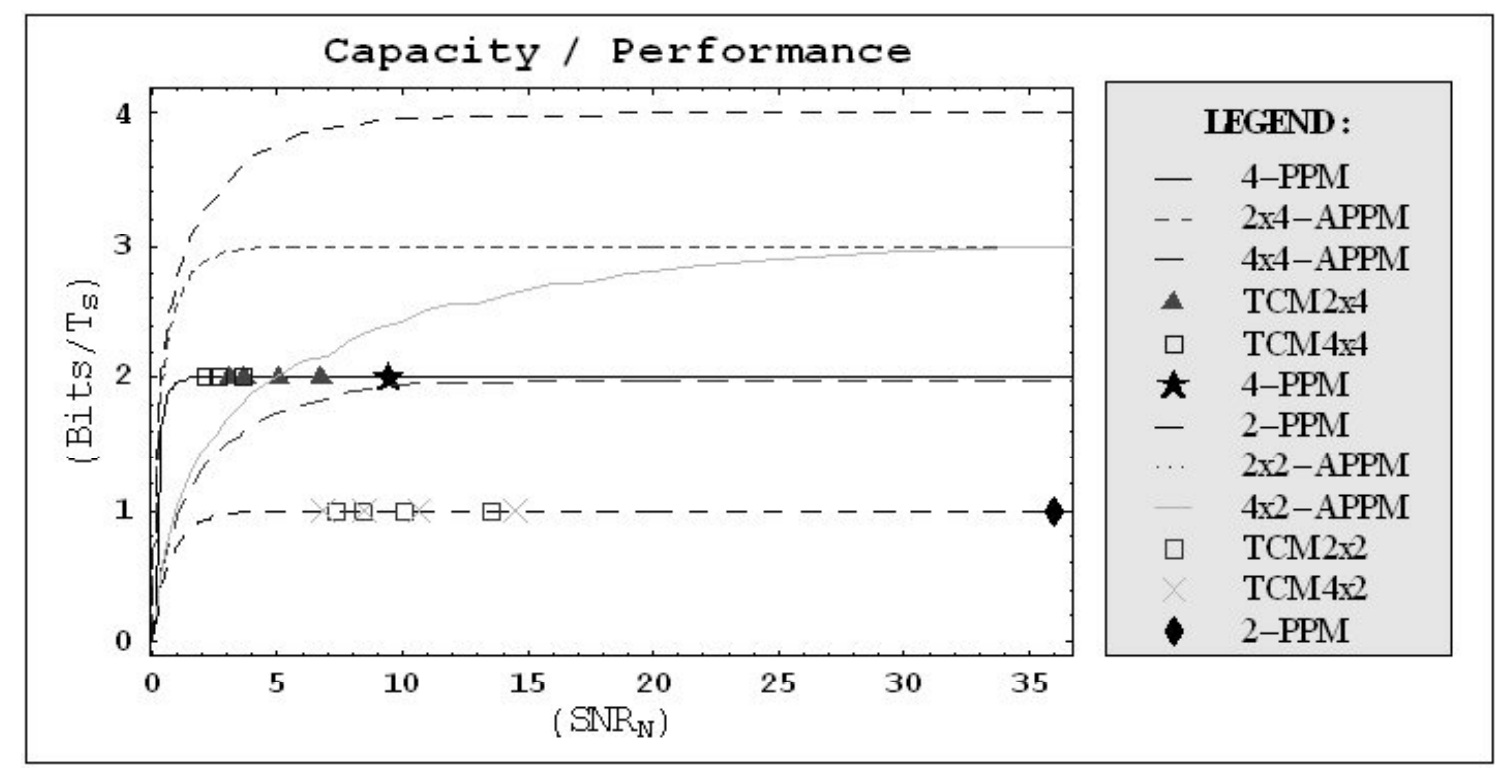

Fig. 4. Marked points represent the required $S N R$ to achieve a $B E R=10^{-9}$ performance for some: uncoded PPM and TCM coded with APPM schemes. Curves represent the respective channel capacities with asymptotic values of 1 and $2 \mathrm{bit} / T_{\text {symbol }}$.

Plots similar to those in Fig. 3 were attained for the same systems operating at two ISI levels with delay spreads per bit duration $\tau_{\text {bit }}=0.06$ and 0.2 . Naturally, when increasing the ISI level the performance of all systems degrade. Even so, in general, the proposed TCM coded systems still maintain the coding gains against the uncoded PPM ones. Fig. 5 plots the required SNR values to achieve the BER $=10^{-6}$ performance versus the simulated values of channel delay spread per bit duration $\tau_{\text {bit }}=\{0,0.06,0.2\}$. The system's performances of our best TCM with PPM based modulations are compared with uncoded PPM and trellis-coded high order PPM proposed by Lee in [10]. Lee considered the use of higher PPM orders expanding the signal-set to accommodate the TCM coding redundancy. Then, in order to minimize the effects of multipath ISI, Lee made a conscious set partitioning in the code design, forming the various partition sets by putting together the symbols whose pulse chips are more separated in time. In a dispersionless channel these aware would be pointless, since the pulse orthogonality in PPM schemes grants the Euclidean distance between any pair of symbols to be the same. Fig. 5 exhibit the way as the several systems performances degrade through ISI level increase, but a straight comparison is impossible once, for a given bit rate, these 


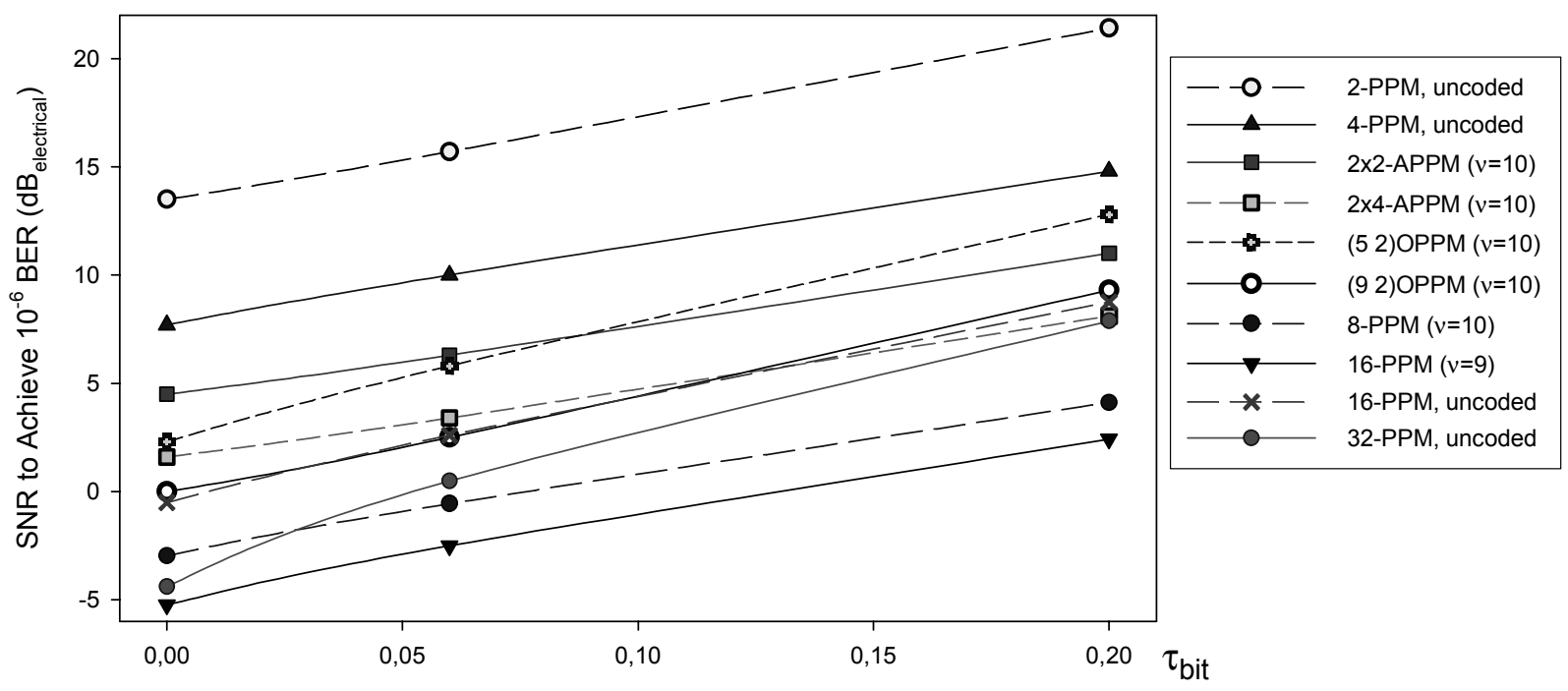

Fig. 5. Simulated SNR requirements to achieve $10^{-6}$ BER versus $\tau_{\text {bit }}=\{0,0.06,0.2\}$, the channel delay spread divided by bit duration, for uncoded PPM systems, high order PPM TCM coded systems [10] and our APPM and OPPM TCM coded systems.

systems hold very different bandwidth requirements. Nonetheless, in general the curves present almost identical slopes which lead us to conclude that, although our codes were derived for AWGN channel (without ISI awareness) their behavior in multipath dispersion channels is quite effective. Another fact arises from Fig. 5 observation, the coded OPPM systems are more affected by the ISI increase than PPM and APPM coded systems. This result does not surprise once, among the schemes with equal alphabet cardinality, the OPPM ones have the narrowest chip durations, what makes them more impaired by ISI. The recent simulation results we got show that better performances can be achieved with appropriated set-partitioning taking in account the ISI effects, as was done in [10], specially for TCM coded OPPM systems.

\section{CONCLUSIONS}

The best codes for some PPM based schemes, such as $\mathrm{A} \times \mathrm{M}-\mathrm{APPM}$ and $(\mathrm{n} \mathrm{w})-\mathrm{OPPM}$, were found and their asymptotic coding gains computed relatively to the uncoded 2-PPM system, for the dispersionless channel case. Monte Carlo simulation results validate the expected coding gains. Thus confirming both APPM and OPPM as quite simple but effective ways of expanding the alphabet size of PPM to apply TCM techniques, and then to improve the performance of wireless infrared communication systems, both in the absence and in the presence of non-severe ISI. As the multipathinduced ISI increases, the more importance the modulation set partitioning take in a TCM code design.

\section{REFERENCES}

[1] J. Kahn, J. Barry, "Wireless Infrared Communications", Proceedings of the IEEE, vol. 85, nº. 2, Feb. 1997.
[2] R. Valadas et al., The Infrared Physical Layer of the IEEE 802.11 Standard for Wireless Local Area Networks, IEEE Communication Magazine, Dec. 1998, 2-7.

[3] S. Williams, IrDA: Past, Present and Future, IEEE Communication Magazine, Feb. 2000, 11-19.

[4] R. Alves, A. Gameiro, "Trellis Codes Based on Amplitude and Position Modulation for Infrared WLAN's", VTC '99-Fall, Vol.5, pg. 29342938, Amsterdam, September 1999.

[5] C. Georghiades, "Some Implications of TCM for Optical DirectDetection Channels", IEEE Trans. on Communications, Vol.37, № 5, May 1989.

[6] R. Alves, A. Gameiro, "Coding of PPM Based Modulation Techniques to Improve the Performance of Infrared WLAN's", VTC '99-Fall, Vol.5, pg. 2934-2938, Amsterdam, September 1999.

[7] S. Benedetto, M. Mondim and G. Montorsi: "Performance Evaluation of Trellis-Coded Modulation Schemes", Proceedings of the IEEE, Vol. 82, $\mathrm{N}^{\circ}$ 6, June 1994.

[8] S. Pietrobon, R. Deng, A. Lafanechére, G. Ungerboeck, D. Costello: "Trellis-Coded Multidimensional Phase Modulation", IEEE Trans. on Information Theory, Vol. 36, № 1, January 1990.

[9] J. B. Carruthers and J. M. Kahn, "Modeling of non-directed wireless infrared channels", in Proc. IEEE Int. Conf. Communications (ICC'96), Dallas, TX, June 1996.

[10] D. Lee, J. Kahn, M. Audeh, "Trellis-Coded Pulse-Position Modulation for Indoor Wireless Infrared Communications", IEEE Transactions On Communications, vol. 45, nº. 9, Sep. 1997. 